\title{
Prognostic Factors for COVID-19 Pneumonia Progression to Severe Symptoms Based on Earlier Clinical Features: A Retrospective Analysis
}

\author{
Huang Huang ${ }^{\dagger}$, Shuijiang Cai ${ }^{\dagger}$, Yueping Li, Youxia Li, Yinqiang Fan, Linghua Li, \\ Chunliang Lei, Xiaoping Tang, Fengyu Hu, Feng Li ${ }^{\text {*f }}$ and Xilong Deng ${ }^{\text {*f }}$ \\ Guangzhou Eighth People's Hospital, Guangzhou Medical University, Guangzhou, China
}

\section{OPEN ACCESS}

Edited by:

Longxiang Su,

Peking Union Medical College

Hospital (CAMS), China

Reviewed by:

Ana Afonso,

University of São Paulo, Brazil

Hongcui Cao,

Zhejiang University, China

*Correspondence:

Feng $\mathrm{Li}$

gz8h_lifeng@126.com

Xilong Deng

gz8hdx|@126.com

${ }^{\dagger}$ These authors have contributed equally to this work

FThese authors have contributed equally to this work and share senior authorship

Specialty section:

This article was submitted to Infectious Diseases - Surveillance,

Prevention and Treatment

a section of the journa

Frontiers in Medicine

Received: 30 April 2020 Accepted: 07 September 2020

Published: 05 October 2020

Citation:

Huang H, Cai S, Li Y, Li Y, Fan Y, Li L, Lei $C$, Tang $X$, Hu F, Li F and Deng $X$ (2020) Prognostic Factors for COVID-19 Pneumonia Progression to Severe Symptoms Based on Earlier Clinical Features: A Retrospective Analysis. Front. Med. 7:557453 doi: 10.3389/fmed.2020.557453
Approximately $15-20 \%$ of COVID-19 patients will develop severe pneumonia, and about $10 \%$ of these will die if not properly managed. Earlier discrimination of potentially severe patients basing on routine clinical and laboratory changes and commencement of prophylactical management will not only save lives but also mitigate the otherwise overwhelming healthcare burden. In this retrospective investigation, the clinical and laboratory features were collected from 125 COVID-19 patients who were classified into mild (93 cases) or severe (32 cases) groups according to their clinical outcomes after 3-7 days post-admission. The subsequent analysis with single-factor and multivariate logistic regression methods indicated that 17 factors on admission differed significantly between mild and severe groups but that only comorbidity with underlying diseases, increased respiratory rate $(>24 / \mathrm{min})$, elevated C-reactive protein (CRP $>10 \mathrm{mg} / \mathrm{L}$ ), and lactate dehydrogenase ( $\mathrm{LDH}>250 \mathrm{U} / \mathrm{L}$ ) were independently associated with the later disease development. Finally, we evaluated their prognostic values with receiver operating characteristic curve (ROC) analysis and found that the above four factors could not confidently predict the occurrence of severe pneumonia individually, though a combination of fast respiratory rate and elevated LDH significantly increased the predictive confidence ( $A \cup C=0.944$, sensitivity $=0.941$, and specificity $=0.902$ ). A combination consisting of three or four factors could further increase the prognostic value. Additionally, measurable serum viral RNA post-admission independently predicted the severe illness occurrence. In conclusion, a combination of general clinical characteristics and laboratory tests could provide a highly confident prognostic value for identifying potentially severe COVID-19 pneumonia patients.

Keywords: COVID-19, SARS-CoV-2, risk factor, clinical manifestation, prognostic factor

\section{BACKGROUND}

The novel coronavirus (SARS-CoV-2) has seemed to sweep across the globe ever since its first successful jump from bat to human being through a still unknown intermediate(s) in approximately late Nov 2019; it still shows a tendency toward significant surges in incidence worldwide (1-3). The SARS-CoV-2 virus seems more contagious than its sibling virus, severe acute respiratory syndrome (SARS) virus, which broke out in 2003; as of March 11, over 120,000 individuals have contracted 
COVID-19 pneumonia within 3 months, which was about 15 times that of the total SARS cases (8,000 in 7 months) (4). The surging increase in COVID-19 patients within a short time window will severely impact the limited medical resources, including physicians, nurses, protective suits, masks, and goggles. Data from the Chinese mainland showed that the majority of total infected patients will recover under simple supervision management, such as quarantine in a compartment hospital isolation ward, but that the overall case fatality rate was $2.3 \%$ (5). For the clinical treatment of COVID-19 patients under shortage of enough medical supplies, the critical issues and priorities are to treat the severe COVID-19 patients [about $20 \%$ of the whole population (5)] and to save their lives with preventive and intensive medical care. However, the clinical presentation of COVID-19 patients differs substantially, and this can include asymptomatic infection, mild upper respiratory tract illness, and severe viral pneumonia (2, 6-8). The most crucial issue is therefore to identify these patients and prioritize their treatment strategy by applying prophylactic medical treatment and management before they progress to the severe stage.

As we know, respiratory function worsens in the severe stage. In clinical practice, saturated oxygen $(<93 \%$ in rest state), reparatory rate ( $>30$ times/min), and deteriorated chest radiology imaging (X-Ray and $\mathrm{CT}$ more high resolution) provide references to confirm their severity $(5,9,10)$. Because of the hypoxia stress, most patients will experience an over-reactivated immune storm, including elevated expression levels of some specific immunological cytokines and changes in certain types of immune cell counts $(6,11)$. Biopsy analysis also showed that the lung bilateral diffuse alveolar damage with cellular fibromyxoid exudates (12). However, CT imaging and immunology detection is not only expensive but also largely unavailable an unable to cope with the significant rise in suspected cases, particularly in those hospitals that are not well-equipped. Can some routine clinical characteristics or/and laboratory measurements (or their combination) predict the occurrence of severe cases?

In this study, we retrospectively analyzed the clinical characteristics of those patients who progressed to severe pneumonia later and found that five simple clinical features and laboratory detection at an earlier time point could serve as prognostic factors facilitating discrimination of severe cases in advance.

\section{METHODS}

\section{Patients}

COVID-19 diagnosis was determined according to the criteria in the new Coronavirus pneumonia diagnosis and treatment plan (trial version 6) issued by the National Health and Health Commission (13). All 298 COVID-19 patients admitted to Guangzhou Eighth People's Hospital from January 20 to February 29, 2020, were included in this study. This study complied with the medical ethics of Guangzhou Eighth People's Hospital. We obtained written consent from the patients.

For this analysis, inclusion criteria were the following: (1) diagnosed as mild or ordinary on admission and (2) length of hospitalization $>3$ days and overall duration of the disease $>7$ days. Qualified patients were then classified into a mild symptom group and a severe symptom group based on the clinical manifestation. The severe symptom diagnosis was determined according to the following criteria: (1) respiratory distress, $R R \geq$ 30 times/min in the resting state; (2) oxygen saturation $\leq 93 \%$ in the resting state; and (3) arterial blood oxygen partial pressure $\left(\mathrm{PaO}_{2}\right)$ /oxygen concentration $\left.\left(\mathrm{FiO}_{2}\right) \leq 300 \mathrm{mmHg}\right)$. The rest of the patients were in the mild group.

\section{Data Collection}

Patient general information, including gender, age, underlying diseases, epidemic history, etc., and their clinical data including symptoms, signs, clinical classification (course duration $>7$ days), laboratory test results, and SARS-CoV-2 viral test results were obtained with standardized data collection forms from electronic medical records.

\section{Statistical Analysis}

Quantitative data was firstly tested to be normality distribution with the Kolmogorov-Smirnov method. Then, for normalized distributed data, $t$-test and Tamhane T2 methods were used for variance of even and uneven data, respectively. For nonnormal data, which was expressed as the median (quartile) [M (P25, P75)], the Mann-Whitney $U$-test was employed. The chi-square test (or Fisher exact probability method) was utilized for analyzing qualitative data. Logistic regression analysis and the receiver operating characteristic curve (ROC) analysis was employed to analyze the independent risk factors. The difference was statistically significant at $P<0.05$. All analysis was performed using SPSS software (version 20.0).

\section{RESULTS}

\section{Patient General Information}

A total of 298 COVID-19 cases (about $85 \%$ of total cases in Guangzhou, China) were admitted to Guangzhou Eighth People's Hospital for treatment from January 20 to February 29, 2020 (Figure 1). According to the inclusion criteria, 173 cases were excluded because 23 cases were already in the severe symptom stage, 52 cases had a short hospitalization time of $<7$ days, and 98 patients had other defects, such as being short of a complete set of detection. Finally, 125 cases, including 63 males and 62 females, were qualified to be included for further investigation, and all their disease courses were over 7 days, with a maximum of 32 days. Based on the severity of disease at 3 days post-admission, 93 patients fell in the mild group ( 38 general cases and 55 mild cases) and 32 patients in the severe group (25 severe cases and seven critical cases).

All included patients were aged between 1.5 and 91 years (averaged $44.87 \pm 18.55$ years) (Table 1). Among them, 37 cases had at least one underlying disease, including 20 cases with hypertension, eight cases with diabetes, five cases with coronary heart disease, two cases with chronic obstructive pulmonary disease, two cases with chronic kidney disease, two cases with chronic liver disease, and two cases with sleep apnea syndrome. Five individuals with two or more basic disorders and 7 cases with obesity (BMI > 26). Epidemiologically, 88 cases had a history of 


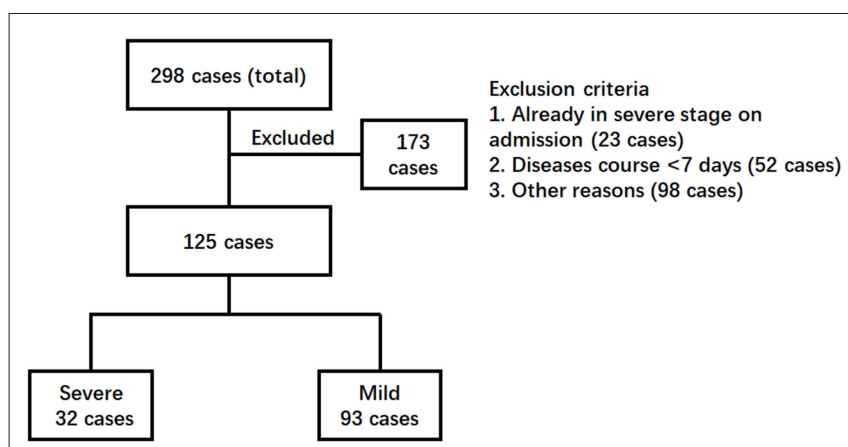

FIGURE 1 | Enrollment chart of COVID-19 patients. The clinical information of a total of 298 patients admitted to the hospital was reviewed. Patients were excluded according to the criteria (1) already in severe stages, (2) with a disease course $<7$ days, and (3) other reasons such as incomplete detection panel. Finally, 125 patients were further divided into two groups. The severe group included the patients who developed severe COVID-19 pneumonia later ( $>3$ days post-admission). The patients remaining were kept in the mild group.

traveling to or living in the Hubei epidemic area before disease onset. Interestingly, we observed that seven patients developed serum SARS-CoV-2 viral RNA positive after admission but ahead of diagnosis to be a severe symptom.

\section{Factors Differed Between the Mild Group and the Severe Group}

The single-factor analysis was applied for each factor between the mild group and the severe group (Table 1). More patients in the severe group were old, obese $($ BMI > 26), and had underlying diseases, particularly hypertension and diabetes $(P$ $<0.05)$, compared with the mild group. Among the general factors, no significant difference could be seen with regards to gender, history of traveling to or living in an epidemic region, coughing, sneezing, muscle joint pain, headache, fatigue, and gastrointestinal symptoms between these two groups $(P>0.05)$. However, more patients in the severe group exhibited high fever, chest tightness, and shortness of breath (fast respiratory rate) $(P<0.05)$. The serum concentration of $\mathrm{C}$-reactive protein, procalcitonin, D-dimer, albumin, and lactate dehydrogenase $(\mathrm{LDH})$ increased significantly in the severe group $(P<0.05)$.

Compared to the mild group, patients in the severe group had lower absolute lymphocyte counts, higher eosinophil counts $(P<0.05)$, and similar levels of other parameters, including white blood cells, neutrophils, platelets, hemoglobin, prothrombin time, activated partial thromboplastin time, blood lactic acid, blood creatinine, and creatine kinase. Interestingly, the levels of glutamate aminotransferase (ALT) and aspartate aminotransferase (AST) significantly increased for severe patients $(P<0.05)$. However, the median values of ALT and AST were still within the normal range, indicating that most of the severe COVID-19 patients had no significant liver damage.

Importantly, all seven patients with the presence of SARSCoV-2 viral RNA in blood during the hospitalization, but before being in the severe stage, finally progressed to the severe stage; they included two severe cases and five critical cases $(P<0.05)$.

\section{Binary Logistic Regression Analysis of COVID-19 Severe Risk Factors}

Next, all categorical variables were converted into covariates, including age, presence of underlying diseases (Yes or No), hypertension (Yes or No), diabetes (Yes or No), obesity (Yes or No), Temperature $\left(<37.4,37.4-38.5,>38.5^{\circ} \mathrm{C}\right)$, fast respiratory rate (Yes or No), elevated C-reactive protein $(>10 \mathrm{mg} / \mathrm{L})$, decreased lymphocyte count $\left(<1.1^{*} 10 \mathrm{E} 9 / \mathrm{L}\right)$ and eosinophil count $\left(<0.02^{*} 10 \mathrm{E} 9 / \mathrm{L}\right)$, elevated procalcitonin $(>0.05 \mathrm{ng} / \mathrm{L})$, elevated D-dimer $(>=2.25 \mu \mathrm{g} / \mathrm{L})$, decreased albumin $(<35 \mathrm{~g} / \mathrm{L})$, and elevated lactate dehydrogenase ( $\mathrm{LDH},>250 \mathrm{U} / \mathrm{L})$, and were then subjected to single-factor logistic regression together with multiple independent variables. Those variables with statistical significance were chosen for subsequent binary logistic regression analysis to test the model coefficients, goodness-of-fit, and multicollinearity. Four factors identified to be significantly relevant to the severity of COVID-19 were underlying diseases (X1), fast respiratory rate ( $>24$ times/min) (X2), elevated Creactive protein level $(\mathrm{CRP}>10 \mathrm{mg} / \mathrm{L})(\mathrm{X} 3)$, and elevated lactate dehydrogenase level (LDH > $250 \mathrm{U} / \mathrm{L})$ (X4) (Table 2). Finally, the multifactor logistic regression equation was obtained: $P=-6.488+2.752 \mathrm{X} 1+4.056 \mathrm{X} 2+2.424 \mathrm{X} 3+5.392 \mathrm{X} 4$. The $\beta$ values and odds ratios (OR) for each factor are shown in Table 2. The result indicated that elevated LDH ranks as having the highest correlation to severe symptom development $(\mathrm{OR}=219.608)$, followed by the fast respiratory rate (OR $=57.726)$, underlying diseases $(\mathrm{OR}=15.67)$, and elevated $\mathrm{CRP}(\mathrm{OR}=11.289)$.

\section{The Prognostic Capacity for Severe Symptom Development}

To better evaluate the prediction capacity of each of the independent risk factors, we plotted their receiver operating characteristic curve (ROC) for the development of severe COVID-19 pneumonia and calculated the area under the ROC curve (AUC value), sensitivity, specificity, Cut-off value, Youden index, and $p$-value (Table 3) for all of them. According to the general standard that AUC values between 0.7 and 0.9 mean a medium level of diagnostic values and AUC values over 0.9 mean a high level of diagnostic values, we observed that all the factors (AUC $<0.9$ ) failed to provide a high prognostic value when used alone. A two-factor combination test then showed that the combination of fast respiratory rate and elevated LDH could provide a highly confident prediction $(\mathrm{AUC}=0.944$, sensitivity $=0.941$, and specificity $=0.902$ ) (Table 3). The AUC values of elevated LDH plus underlying diseases or plus elevated CRP were both over 0.9 , but their sensitivity or specificity was lower than 0.9 . Then, triple factor combination significantly increased the prognostic efficacy, and all combinations had increased sensitivity and specificity (Table 3). Finally, we calculated the prognostic value of the combination of all four factors and found that the AUC value was significantly increased to 0.985 (95\% CI $0.968-1.000)$, the sensitivity to 0.912 , and the specificity to 0.957 (Table 3). 
TABLE 1 | Characteristics of COVID-19 patients.

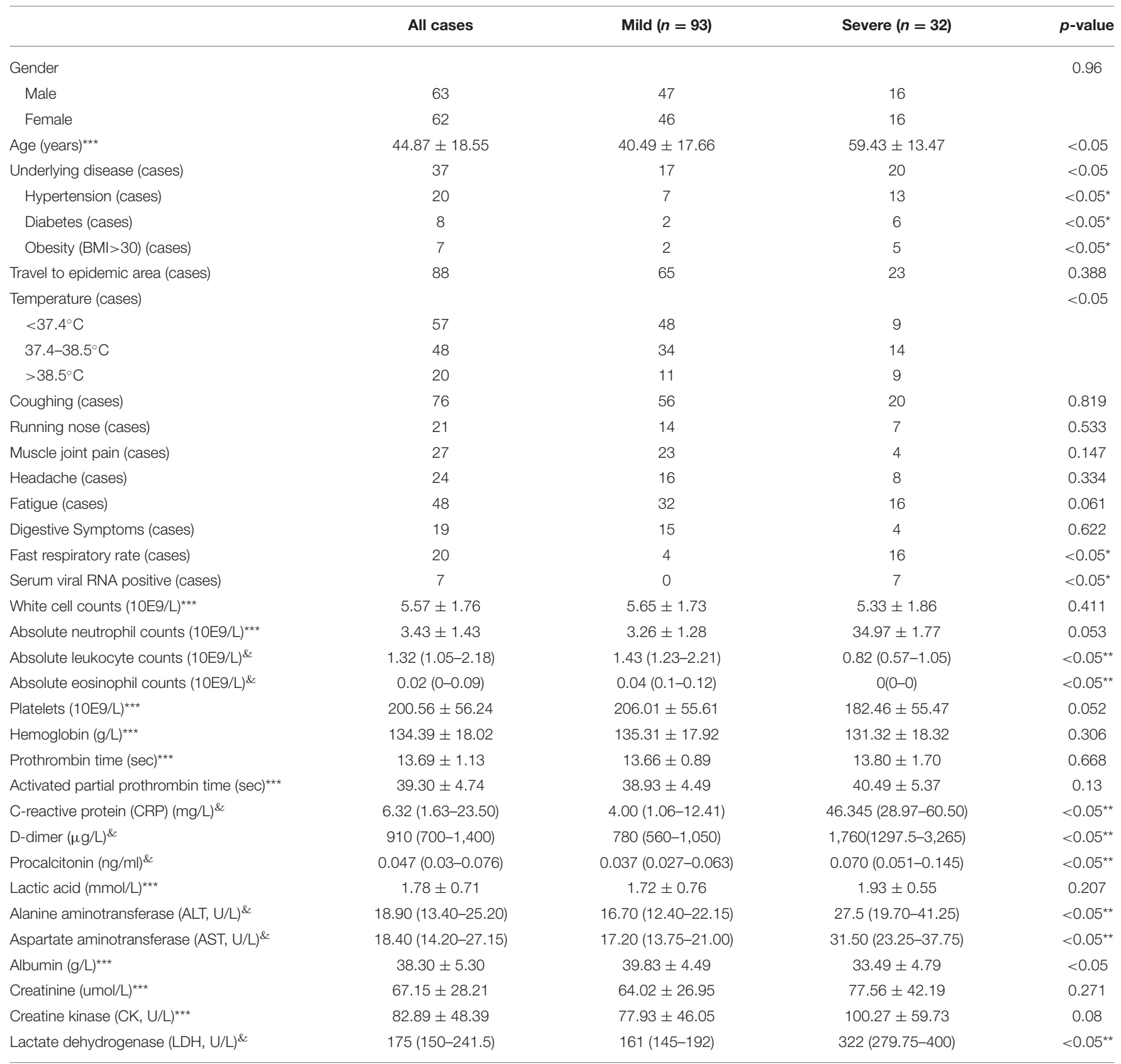

${ }^{\star}$ Fisher's Exact Test.

**Mann-Whitney U-Test.

${ }^{\star * \star}$ average \pm standard deviation (STD).

\& average (95\% confidence interval).

\section{DISCUSSION}

Our study showed that underlying disease, fast respiratory rate (>24 times/min), elevated serum C-reactive protein level (CRP, $>10 \mathrm{mg} / \mathrm{L}$ ), and elevated lactate dehydrogenase level (LDH, $>250 \mathrm{U} / \mathrm{L}$ ) were four independent risk factors for predicting the progression of some COVID-19 patients from mild to severe conditions. Firstly, elevated lactate dehydrogenase levels ranked as number $1(\mathrm{OR}=219.332)$ and fast respiratory rate as number $2(\mathrm{OR}=57.726)$ among the four factors (Table 2). Interestingly, an elevated lactate dehydrogenase level was associated with severe SARS infection (14), which broke out in 2003, but was absent in the severe MERS infection (15), which is still circulating. When used individually, all four factors have a moderate prediction value for their low specificity and sensitivity (AUC values < 0.9) (Table 3). Secondly, we found that the combination of two 
TABLE 2 | Independent factors associated with severe symptom development in COVID-19 patients.

\begin{tabular}{|c|c|c|c|c|c|c|}
\hline Variables & $\beta$ & S.E. & chi-square & $P$-value & OR ( $95 \%$ confidence interval) & \\
\hline$x_{1}$ & Underlying disease & 2.752 & 1.066 & 6.666 & 0.01 & $15.67(1.94-126.55)$ \\
\hline$x_{2}$ & Fast respiratory rate (>24 times/min) & 4.056 & 1.183 & 11.76 & 0.001 & $57.726(5.685-586.191)$ \\
\hline$x_{3}$ & CPR (>10 mg/L) & 2.424 & 1.004 & 5.823 & 0.016 & 11.289 (1.577-80.838) \\
\hline$X_{4}$ & $\mathrm{LDH}(>250 \mathrm{U} / \mathrm{L})$ & 5.392 & 1.24 & 18.911 & $<0.001$ & 219.608 (19.332-2494.742) \\
\hline Intercept & & -6.488 & 1.499 & 18.738 & $<0.001$ & 0.002 \\
\hline
\end{tabular}

S.E., standard error;

OR (95\% Cl), Odd Ratio (95\% confidence interval).

TABLE 3 | Prognostic values for severe COVID-19 pneumonia development.

\begin{tabular}{|c|c|c|c|c|c|c|c|}
\hline & Factor & AUC (95\% Cl) & Sensitivity & Specificity & Cut-off value & Youden Index & $P$-value \\
\hline \multirow[t]{4}{*}{ Single factor } & Underlying diseases (1) & $0.722(0.614-0.829)$ & 0.618 & 0.826 & 0.367 & 0.444 & $<0.001$ \\
\hline & Fast respiratory rate (2) & $0.758(0.648-0.867)$ & 0.559 & 0.957 & 0.492 & 0.516 & $<0.001$ \\
\hline & Elevated CRP (3) & $0.774(0.685-0.864)$ & 0.853 & 0.696 & 0.298 & 0.549 & $<0.001$ \\
\hline & Elevated LDH (4) & 0.855 (0.766-0.944) & 0.765 & 0.946 & 0.461 & 0.711 & $<0.001$ \\
\hline \multirow[t]{6}{*}{ Two factors } & $(1)+(2)$ & 0.853 (0.767-0.939) & 0.824 & 0.793 & 0.223 & 0.617 & $<0.001$ \\
\hline & $(1)+(3)$ & 0.854 (0.779-0.928) & 0.853 & 0.696 & 0.274 & 0.549 & $<0.001$ \\
\hline & $(1)+(4)$ & $0.940(0.894-0.987)$ & 0.971 & 0.783 & 0.156 & 0.754 & $<0.001$ \\
\hline & $(2)+(3)$ & $0.870(0.795-0.944)$ & 0.912 & 0.663 & 0.18 & 0.575 & $<0.001$ \\
\hline & $(2)+(4)$ & $0.944(0.892-0.996)$ & 0.941 & 0.902 & 0.315 & 0.843 & $<0.001$ \\
\hline & $(3)+(4)$ & $0.918(0.856-0.981)$ & 0.765 & 0.946 & 0.365 & 0.711 & $<0.001$ \\
\hline \multirow[t]{4}{*}{ Three factors } & $(1)+(2)+(3)$ & 0.910 (0.850-0.969) & 0.765 & 0.902 & 0.253 & 0.667 & $<0.001$ \\
\hline & $(1)+(2)+(4)$ & $0.976(0.955-0.998)$ & 0.912 & 0.935 & 0.411 & 0.846 & $<0.001$ \\
\hline & $(1)+(3)+(4)$ & 0.963 (0.933-0.993) & 0.912 & 0.891 & 0.227 & 0.803 & $<0.001$ \\
\hline & $(2)+(3)+(4)$ & $0.964(0.919-1.000)$ & 0.912 & 0.934 & 0.355 & 0.847 & $<0.001$ \\
\hline Four factors & $(1)+(2)+(3)+(4)$ & 0.985 (0.968-1.000) & 0.912 & 0.957 & 0.374 & 0.869 & $<0.001$ \\
\hline
\end{tabular}

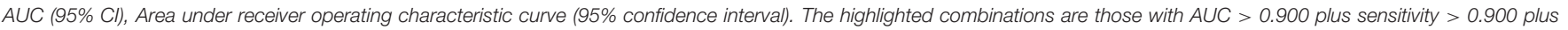
specificity $>0.900$, which indicates significant and reliable prognostic values for clinical practice.

factors, fast respiratory rate plus elevated $\mathrm{LDH}$, could provide a high prognostic value for severe symptom development (AUC $=$ 0.944 , sensitivity $=0.941$, and specificity $=0.902)$. Combinations of triple factors could significantly increase the prognostic value (AUC > 0.9). Finally, a combination of all four factors, provide an excellent prognostic efficacy, achieving AUC $=0.985$ (95\% CI $0.968-1.000)$ with high sensitivity (0.953) and specificity (0.968).

Our hospital has treated over $80 \%$ of COVID-19 patients in Guangzhou city--298 cases as of February 29, 2020-including 55 severe cases but only one death case. All the patients except two patients recovered as of March 15. A retrospective analysis of all the cases revealed that the extremely low fatality rate in our hospital, one of 298 cases $(0.0336 \%)$ - significantly lower than the overall fatality rate $(2.3 \%)$ in China (5), was largely attributed to the effect of an expert panel, consisting of physicians from multiple disciplines, including infectious diseases, respiratory diseases, and intensive care unit (ICU), and radiology. Patients newly admitted were reviewed by the panel, and patients who meet several of the following criteria were transferred immediately to the ICU isolation ward for close supervision, including, (1) the illness onset has entered 7-10 days; (2) over 50 years old; (3) obesity, pregnant women, children; (4) with underlying diseases, especially hypertension, diabetes, COPD; (5) fast respiratory rate; (6) obvious decline in spirit and appetite; (7) significant reduction and/or progressive decline of peripheral blood lymphocytes; (8) decrease in albumin; (9) elevated Creactive protein; (10) elevated lactate dehydrogenase; and (11) quickly deteriorated or with two or more lesions in lungs revealed by chest imaging. Once they progressed to the severe stage, they received treatment immediately. The above four prognostic factors, as routine and affordable clinical characteristics, were included in these criteria, and their immediate and preventive therapies were facilitated retrospectively.

All seven patients who were detected to be serum viral RNA positive developed severe symptoms very soon, which further confirmed our previous observation that detectable 2019-nCoV viral RNA in blood is a reliable indicator for further clinical severity (16). However, as the viral RNA positive rate was low high (seven of 32 cases, $21.8 \%$ ) in this study and other reports (17) and viral RNA detection is expensive, we do not recommend the continuous detection of viral RNA. In this regard, we suggest reserving the precious reagent for confirming virus infection.

In conclusion, our study indicated that underlying disease, a fast respiratory rate, elevated serum C-reactive protein level, and elevated lactate dehydrogenase level significantly correlated to the development of severe COVID-19 pneumonia; 
additionally, elevated lactate dehydrogenase and a fast respiratory rate (possibly plus one or two more other factors) can serve as prognostic factors for the discriminating potential severe cases among the mild COVID-19 patients. Our study provided convenient, reliable, and affordable references for both patients and physicians to make a highly confident decision to commence management and treatment safely.

\section{SUMMARY}

With our successful experience of treating COVID-19 patients, we retrospectively found that routine clinical features could reliably predict severe pneumonia development and could thus provide quick and affordable references for physicians to save patients with otherwise fatal COVID-19 using their limited medical resource.

\section{DATA AVAILABILITY STATEMENT}

The original contributions presented in the study are included in the article/supplementary material, further inquiries can be directed to the corresponding authors.

\section{ETHICS STATEMENT}

Ethical review and approval was not required for the study on human participants in accordance with the local legislation

\section{REFERENCES}

1. World Health Organization. Coronavirus Disease (COVID-2019) Situation Reports. Available online at: https://www.who.int/emergencies/diseases/ novel-coronavirus-2019/situation-reports/

2. Chen N, Zhou M, Dong X, Qu J, Gong F, Han Y, et al. Epidemiological and clinical characteristics of 99 cases of 2019 novel coronavirus pneumonia in Wuhan, China: a descriptive study. Lancet. (2020) 395:507-13. doi: 10.1016/S0140-6736(20)30211-7

3. Li Q, Guan X, Wu P, Wang X, Zhou L, Tong Y, et al. Early transmission dynamics in Wuhan, China, of novel coronavirus-infected pneumonia. N Engl J Med. (2020) 382:1199-207. doi: 10.1056/NEJMoa2001316

4. Wu A, Niu P, Wang L, Zhou H, Zhao X, Wang W, et al. Mutations, Recombination and Insertion in the Evolution of 2019-nCoV. bioRxiv [Preprint]. (2020). doi: 10.1101/2020.02.29.971101

5. Team TNCPERE. Vital surveillances: the epidemiological characteristics of an outbreak of 2019 novel coronavirus diseases (COVID-19) - China, 2020. China CDC Weekly. (2020) 2:113-22. doi: 10.46234/ccdcw2020.032

6. Huang C, Wang Y, Li X, Ren X, Zhao J, Hu Y, et al. Clinical features of patients infected with 2019 novel coronavirus in Wuhan, China. Lancet. (2020) 395:497-506. doi: 10.1016/S0140-6736(20)30183-5

7. Wang D, Hu B, Hu C, Zhu F, Liu X, Zhang J, et al. Clinical characteristics of 138 hospitalized patients with 2019 novel coronavirus-infected pneumonia in Wuhan, China. JAMA. (2020) 323:1061-9. doi: 10.1001/jama.2020.1585

8. Guan WJ, Ni ZY, Hu Y, et al. Clinical characteristics of coronavirus disease 2019 in China. $N$ Engl J Med. (2020) 382:1708-20. doi: 10.1056/NEJMoa2002032

9. Xu X, Yu C, Qu J, Zhang L, Jiang S, Huang S, et al. Imaging and clinical features of patients with 2019 novel coronavirus SARS-CoV-2. Eur J Nucl Med Mol Imaging. (2020) 47:1275-80. doi: 10.1007/s00259-020-04735-9

10. Pan Y, Guan H, Zhou S, Wang Y, Li Q, Zhu T, et al. Initial CT findings and temporal changes in patients with the novel coronavirus pneumonia and institutional requirements. The patients/participants provided their written informed consent to participate in this study.

\section{AUTHOR CONTRIBUTIONS}

$\mathrm{HH}, \mathrm{FL}$, and $\mathrm{XD}$ conceived the study and wrote the manuscript. $\mathrm{HH}$ and SC collected data and performed the data analysis. $\mathrm{HH}$, SC, YuL, YoL, YF, and XD participated in the clinical treatment. LL, CL, and XT supervised the clinical treatment. FH analyzed the results. All authors read the manuscript and approved the final version. All authors contributed to the article and approved the submitted version.

\section{FUNDING}

This work was supported by National Natural Science Foundation of China (Nos. 81670536 and 81770593) and the Chinese National Grand Program on Key Infectious Disease Control (2017ZX10202203-004-002 and 2018ZX10301404-003-002).

\section{ACKNOWLEDGMENTS}

The authors would like to thank all nurses in the treatment team for taking care of the patients and doctors in the expert panel for their treatment guidance. This manuscript has been released as a preprint at medRxiv (18).

(2019-nCoV): a study of 63 patients in Wuhan, China. Eur Radiol. (2020) 30:3306-9. doi: 10.1007/s00330-020-06731-x

11. Zhou Y, Fu B, Zheng X, Wang D, Zhao C, Qi Y, et al. Aberrant pathogenic GM-CSF+ T cells and inflammatory CD14+CD16+ monocytes in severe pulmonary syndrome patients of a new coronavirus. bioRxiv [Preprint]. (2020). doi: 10.1101/2020.02.12.945576

12. Xu Z, Shi L, Wang Y, Zhang J, Huang L, Zhang C, et al. Pathological findings of COVID-19 associated with acute respiratory distress syndrome. Lancet Respir Med. (2020) 8:420-2. doi: 10.1016/S2213-2600(20) 30076-X

13. nhc.gov.cn. Notice on Issuing a New Coronary Virus Pneumonia Diagnosis and Treatment Plan (Trial Version 6, in Chinese). Available online at: http://www. nhc.gov.cn/yzygj/s7653p/202002/8334a8326dd94d329df351d7da8aefc2. shtml

14. Liu CL, Lu YT, Peng MJ, Chen PJ, Lin RL, Wu CL, et al. Clinical and laboratory features of severe acute respiratory syndrome vis-a-vis onset of fever. Chest. (2004) 126:509-17. doi: 10.1378/chest.126.2.509

15. Ko JH, Park GE, Lee JY, Lee JY, Cho SY, Ha YE, et al. Predictive factors for pneumonia development and progression to respiratory failure in MERS-CoV infected patients. J Infect. (2016) 73:468-75. doi: 10.1016/j.jinf.2016.08.005

16. Chen W, Lan Y, Yuan X, Deng X, Li Y, Cai X, et al. Detectable 2019 $\mathrm{nCoV}$ viral RNA in blood is a strong indicator for the further clinical severity. Emerg Microbes Infect. (2020) 9:469-73. doi: 10.1080/22221751.2020. 1732837

17. Wang W, Xu Y, Gao R, Lu R, Han K, Wu G, et al. Detection of SARS-CoV-2 in different types of clinical specimens. JAMA. (2020) 323:1843-4. doi: 10.1001/jama.2020.3786

18. Huang H, Cai S, Li Y, Li Y, Fan Y, Li L, et al. Prognostic factors for COVID-19 pneumonia progression to severe symptom based on the earlier clinical features: a retrospective analysis. medRxiv [Preprint]. doi: 10.1101/2020.03.28.200 45989 
Disclaimer: The contents of this article are solely the responsibility of the authors and do not necessarily represent the views of any organization

Conflict of Interest: The authors declare that the research was conducted in the absence of any commercial or financial relationships that could be construed as a potential conflict of interest.
Copyright (C) 2020 Huang, Cai, Li, Li, Fan, Li, Lei, Tang, Hu, Li and Deng. This is an open-access article distributed under the terms of the Creative Commons Attribution License (CC BY). The use, distribution or reproduction in other forums is permitted, provided the original author(s) and the copyright owner(s) are credited and that the original publication in this journal is cited, in accordance with accepted academic practice. No use, distribution or reproduction is permitted which does not comply with these terms. 